\title{
KORN'S INEQUALITIES FOR GENERALIZED EXTERNAL CUSPS
}

\author{
GABRIEL ACOSTA AND IGNACIO OJEA
}

\begin{abstract}
In this paper we consider a general class of external cusps defined by linking appropriate collections of John domains. For that class, weighted Korn inequalities are proved by means of rather elementary arguments.
\end{abstract}

\section{INTRODUCTION}

For a given a domain $\Omega \subset \mathbb{R}^{n}$ and a vector field $u \in W^{1,2}(\Omega)$, Korn's inequality states

$$
\|D u\|_{L^{2}(\Omega)^{n \times n}} \leq C\|\varepsilon(u)\|_{L^{2}(\Omega)^{n \times n}},
$$

where $D u$ is the differential matrix of $u$ and $\varepsilon(u)$ its symmetric part

$$
\varepsilon(u)_{i, j}=\frac{1}{2}\left(\frac{\partial u_{i}}{\partial x_{j}}+\frac{\partial u_{j}}{\partial x_{i}}\right) .
$$

Inequality (1.1) introduced in [1,2] has become a classic subject in the literature of continuum mechanics. In elasticity theory, $u$ plays the role of the displacement field of an elastic body. In this case $\varepsilon(u)$ is called the linearized strain tensor and (1.1) equals to the coercivity of the bilinear form associated to the underlying linear equations.

Non-constant vector fields in the kernel of $\varepsilon$ can not obey (1.1) and therefore Korn's inequality can not hold without considering some extra conditions. Two classic cases treated in the seminal works by Korn and called the first and the second case of the inequality state that (1.1) holds if either $u$ vanishes on the boundary of $\Omega$ or if

$$
\int_{\Omega} \frac{D u-D u^{t}}{2}=0
$$

respectively. The first case can be proved by means of very simple arguments and as it is well known it holds for any bounded domain. On the other hand, the second case requires deeper considerations and actually it fails for domains with poor regularity.

Inequality (1.1) can be found in different forms involving traditional and weighted spaces. In $L^{p}$ norm it reads

$$
\|D u\|_{L^{p}(\Omega)^{n \times n}} \leq C\|\varepsilon(u)\|_{L^{p}(\Omega)^{n \times n}},
$$

where $p$ should be in the range $1<p<\infty$. Another version, sometimes called the general case of Korn's inequality, takes the form

$$
\|D u\|_{L^{p}(\Omega)^{n \times n}} \leq C\left\{\|u\|_{L^{p}(\Omega)^{n}}+\|\varepsilon(u)\|_{L^{p}(\Omega)^{n \times n}}\right\},
$$

in which no extra conditions other than $u \in W^{1, p}(\Omega)^{n}$ are required. It can be shown, see e.g. [3], that (1.3) implies (1.4) for any domain $\Omega$. On the other hand, for regular domains (1.3) can be deduced from (1.4) using compactness arguments [4].

\footnotetext{
Key words and phrases. Poincaré inequality, Korn inequality, elasticity, external cusps, weighted Sobolev spaces.

This work was partially supported by ANPCyT under grant PICT 2010-1675. The first author is a member of the IMAS-CONICET and the second author is a fellow of CONICET.
} 
Many proofs of Korn's inequality have been given since Korn's original works, and even a short review of this subject would involve a large number of references. Friederichs [5] was unable to reproduce Korn's arguments for the second case and proved the inequality for smooth domains by reducing the second to the first case. Since then, different arguments allowed to treat less regular domains. That is the case of those arguments involving singular integrals (see [4] and the references therein) where typically the derivatives of the vector field $u$ are written as an average of derivatives of $\varepsilon(u)$ in a cone. This idea, that follows closely Calderón's extension method, applies naturally for Lipschitz domains since they enjoy the cone property. In this regard, let us recall that the connection of Korn's inequality with extension procedures was also exploited by Nitsche, who used elementary arguments [6] to prove (1.1), in the second case, for Lipschitz domains by modifying appropriately the extension operator due to Stein. The same line of reasoning is applied in [7], where the authors prove that (1.4) stands not only for Lipschitz but for the broader class of uniform domains, using a modification of the extension operator given by Jones in [8]. In spite of these results let us observe that the second case of (1.3) holds for

$$
\Omega_{\infty}=B(0,1) \backslash\left\{(x, y) \in \mathbb{R}^{2}: 0 \leq x \leq 1, y=0\right\},
$$

where $B(0,1)$ is the unitary ball. However it is easy to see that $\Omega_{\infty}$ is not an extension domains. Therefore it becomes clear that extension arguments should fail to tackle (1.3) in the more general setting. Actually, (1.3) holds in the second case for a family of domains that contains strictly the set of uniform domains. In this concern let us recall the class of domains introduced by John in [9], and named John domains after him by Martio and Sarvas [10].

Definition 1.1. Let $0<\alpha \leq \beta<\infty$. A domain $\Omega \subset \mathbb{R}^{n}$ is called a John domain with parameters $\alpha$ and $\beta$ if there is a point $x_{0} \in \Omega$ (the John-center of $\Omega$ ) such that for every $x \in \Omega$ there is a rectifiable curve with parametrization by arc length $\gamma:[0, \ell] \rightarrow \Omega$ such that $\gamma(0)=x$ and $\gamma(\ell)=x_{0}$, and:

$$
\begin{gathered}
\ell \leq \beta, \\
d(\gamma(t), \partial \Omega) \geq \frac{\alpha}{\ell} t \quad \forall t \in[0, \ell] .
\end{gathered}
$$

Given $x \in \Omega$, and its correspondant curve $\gamma$, the set $\cup_{t} B\left(\gamma(t), \frac{\alpha}{\ell} t\right) \subset \Omega$ can be regarded as a twisted cone with its axis depicted by the curve $\gamma$. In this sense we may say that in a John domain $\Omega$ any $x \in \Omega$ can be joined with $x_{0}$ through a curve that remains "away" from the boundary. John domains contains star-shaped, Lipschitz and Uniform domains. The inner cusp

$$
\Omega_{\alpha}=B(0,1) \backslash\left\{(x, y) \in \mathbb{R}^{2}:|y|<x^{\alpha}\right\} \quad \alpha>1,
$$

as well as the limit case (1.5) are examples of John domains. The boundary of a John domain can be very intricate, for instance the Koch snowflake, with fractal boundary is also a John domain. In Figure 1, we show all these examples along with schematic twisted cones. Korn's inequality holds on John domains, with a constant depending only on the parameters $\alpha$ and $\beta$. This is shown in [11] where an explicit continuous right inverse of the divergence is constructed. In simple geometries it is possible to get more information about the constant involved in the inequality. For instance, in a convex domain $\Omega$ the constant depends linearly on the ratio between the diameter of $\Omega$ and the diameter of a maximal ball contained in $\Omega$ [12]. Remarkably, for star-shaped domains the same bound is valid in dimension two as it is shown in [13]. For star-shaped domains, the dependance of the constant on that ratio in dimension $n$ is also studied in $[12]$.

Although Korn's inequality holds for very general domains it fails, as it was early noticed by Friederichs, in domains with external cusps (see [14] for a collection of counterexamples). Roughly speaking, an external cusp is a domain that narrows towards a point (the tip of the 


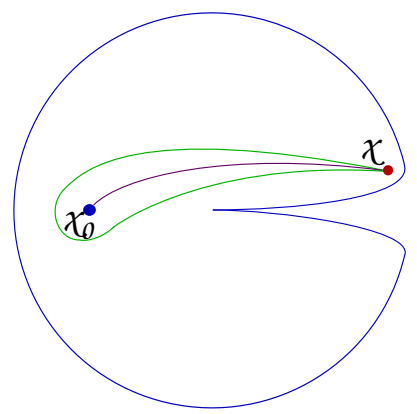

(A) The inner cusp $\Omega_{\alpha}$

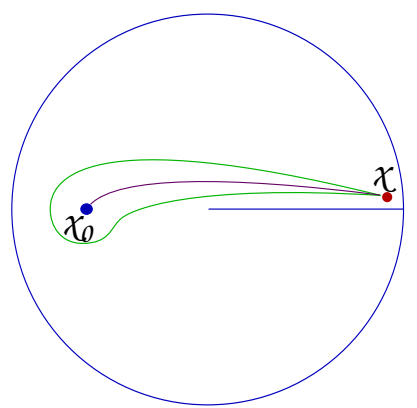

(в) The limit case $\Omega_{\infty}$

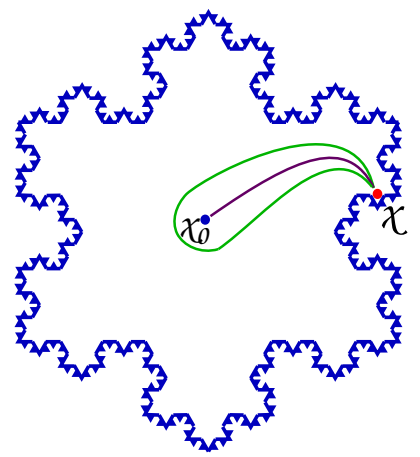

(c) The Koch snowflake

FiguRE 1. Examples of John domains.

cusp) faster than any cone. Observe that this narrowing prevents any external cusp to be a John domain.

The simplest kind of external cusps are given by power type cusps:

$$
\Omega=\left\{\left(x^{\prime}, x_{n}\right) \in \mathbb{R}^{n-1} \times \mathbb{R}:\left|x^{\prime}\right|<x_{n}^{\gamma}\right\},
$$

being $\gamma>1$ a real number. This notion is naturally generalized to domains with a profile depicted by a more general function $\varphi$ :

$$
\Omega=\left\{\left(x^{\prime}, x_{n}\right) \in \mathbb{R}^{n-1} \times \mathbb{R}:\left|x^{\prime}\right|<\varphi\left(x_{n}\right)\right\},
$$

where $\varphi: \mathbb{R}_{\geq 0} \longrightarrow \mathbb{R}_{\geq 0}$ is a non-decreasing derivable function such that $\varphi(0)=0$ and $\varphi^{\prime}(0)=0$, or, more generally, $\varphi$ is non-decreasing, Lipschitz and $\frac{\varphi(t)}{t} \longrightarrow 0\left(t \longrightarrow 0^{+}\right)$.

The main precedents that we follow regarding Korn's inequality on external cusps work with these definitions. In [15] power type cusps are treated, and the existence of a right inverse for the divergence operator in weighted spaces is proved. As a corollary, the following weighted Korn's inequality is obtained (see [15, Theorem 6.2]):

Theorem A. Given $\Omega$ a domain of the form (1.9), $1<p<\infty, B \subset \Omega$ an open ball and $\beta \geq 0$; there exists a constant $C$, depending only on $\Omega, B, p$ and $\beta$, such that for every $u \in W_{d^{p \beta}}^{1, p}(\Omega)^{n}$ :

$$
\|D u\|_{L_{d p \beta}^{p}(\Omega)^{n \times n}} \leq C\left\{\|u\|_{L^{p}(B)^{n}}+\|\varepsilon(u)\|_{L_{d^{p}(\beta+1-\gamma)}^{p}(\Omega)^{n \times n}}\right\},
$$

where $d=d(x)$ is the distance to the origin and $\gamma$ is the exponent of the cusp.

Notice that for $W^{1, p}(\Omega)^{n}$ (i.e. $\beta=0$ ) the weight on the right hand side, due to the cuspidal behavior of $\Omega$ is $d^{p(1-\gamma)}$. The optimality of Theorem $\mathrm{A}$ is treated in [14], where the authors work with cusps with a general profile $\varphi$ and prove the following theorem:

Theorem B. Let $\Omega$ be a cusp with profile $\varphi$, according to (1.10), $\beta_{1}, \beta_{2} \in \mathbb{R}, 1<p<\infty$ and $B$ a ball compactly contained in $\Omega$. If there is a constant $C$ such that:

$$
\|D v\|_{L_{\left(\varphi^{\prime}\right) \beta^{p \beta_{1}}}^{p}(\Omega)^{n \times n}} \leq C\left\{\|v\|_{L^{p}(B)^{n}}+\|\varepsilon(v)\|_{L_{\left(\varphi^{\prime}\right)^{p \beta_{2}}}^{p}(\Omega)^{n \times n}}\right\},
$$

for every $v \in W_{\left(\varphi^{\prime}\right)^{p \beta_{1}}}^{1, p}(\Omega)^{n}$, then $\beta_{1} \geq \beta_{2}+1$.

Observe that for a power type cusp $\varphi^{\prime}(t)=\gamma t^{\gamma-1}$, and since inside the cusp one has $d(x) \sim x_{n}$, then Korn's inequality in Theorem A corresponds with the case $\beta_{1}=\beta_{2}+1$ in Theorem B, which shows that Theorem A is sharp. 
Other results about external cusps can be found in [16], where weighted anisotropic Korn inequalities for power type cusps are proved in $\mathbb{R}^{3}$. There, the author mentions that more general cases could be treated by using the same arguments developed in that paper.

Let us recall that for a Hölder- $\alpha$ domain weighted versions of Korn's inequality can be found in [17]. Since such a domain may have many "cuspidal" singularities, the weight that naturally arises depends on the distance to the whole boundary. On the other hand, for more general domains abstract weighted Korn inequalities can be derived from results concerning the existence of a right inverse of the divergence operator, see for instance [18], [19].

The aim of the present paper is to extend Theorem A to a wider class of external cusps. Actually, we prove that such a result can be generalized to external cusps whose boundary is locally the one of a John domain. In this context we show that the function $\varphi$ does not need to depict the precise profile of the domain, but only to give a qualitative description of the narrowing.

Our technique is based on rather simple ideas. Since the behavior of the constant in Korn's inequality on rectangles is known, we consider chains of rectangles, and use a discrete Hardy inequality to pass from one rectangle to another, proving a weighted Korn inequality for the whole chain. The weight is piecewise constant and it is given by Korn's constant on each rectangle. This approach depends exclusively on the existence of intermediate rectangles that link each rectangle in the chain with its two neighbors. This approach can be applied to more general subdomains and in this regard we introduce the notion of quasi-rectangle. A quasi-rectangle is, essentially, a domain that contains a rectangle and where Korn and Poincaré inequalities hold with constants similar to those corresponding to this interior rectangle. The boundary of a quasi rectangle can be locally as general as the one of a John domain. The results obtained for chains of rectangles are straightforwardly generalized to chains of quasi-rectangles, yielding weighted Korn's inequalities for a very general class of domains. Finally, we consider as an example, external cusps formed by chains of quasi-rectangles. This chains are defined by requiring an appropriate narrowing towards a "singular" point. In this way our generalization of Theorem A is a simple corollary of our results for chains of quasi-rectangles. Counterexamples proposed in [14] can be easily adapted for proving the optimality of the weights obtained in this paper.

Finally, before proceeding, let us mention that the extension procedure for external cusps used in [20] can be appropriately combined with the extension operator for uniform domains constucted in [7]. In that way it is possible to obtain similar results to those given here. However, by doing that, the notion of "locally John" should be replaced by the notion of "locally uniform" proposed in [20]. This allows to handle a less general class of external cusps than that treated here. This limitation is not surprising in the light of the facts mentioned above concerning the use of extension arguments in the context of Korn's inequality. For that reason we do not follow this approach.

\section{Notation and Preliminaries}

Throughout this article $\hat{x}_{n}$ stands for the $x_{n}$ axis, and $C$ denotes a generic constant that may change from line to line. We say that two positive numbers $a$ and $b$ are $C$-comparable, and we write $a \underset{C}{\sim} b$, if $\frac{1}{C} a \leq b \leq C a$. For every collection of sets $\mathcal{C}$, we denote with $\cup \mathcal{C}$ the union of all the sets in $\mathcal{C}$, i.e. $\cup \mathcal{C}:=\cup_{S \in \mathcal{C}} S$. Given two sets, we write $A \equiv B$ if they differ in measure zero.

In this work we deal with open rectangles $R \subset \mathbb{R}^{n}$ with edges parallels to the coordinate axes. The size vector of $R$ is denoted with $\vec{\ell}(R)=\left(\ell_{1}(R), \ell_{2}(R), \ldots, \ell_{n}(R)\right)$, where $\ell_{i}(R)$ is the length of the $R$ 's i-th edge. For a cube $Q$ we use $\ell(Q)$ to denote the length of any of its edges, and for a rectangle $R$ we take: $L_{M}(R):=\max _{1 \leq i \leq n}\left\{\ell_{i}(R)\right\}$ and $L_{m}(R):=\min _{1 \leq i \leq n}\left\{\ell_{i}(R)\right\}$. In some point we restrict our attention to rectangles with $n-1$ short edges of equal size that 
we denote $\ell(R)$ and a long edge (the vertical) $L(R)$. A pair of rectangles $R_{1}, R_{2}$ are called $C$-comparable, and we write $R_{1} \underset{C}{\widetilde{R}} R_{2}$, if $\ell_{i}\left(R_{1}\right) \underset{C}{\widetilde{C}} \ell_{i}\left(R_{2}\right)$ for $1 \leq i \leq n$. For a rectangle $R$, we denote its center with $c_{R}$. If $c_{R}=\left(c_{1}, \cdots, c_{n}\right)$ the upper face $F_{R}^{u}$ of $R$ is given by $F_{R}^{u}=\left\{\left(x_{1}, \cdots, x_{n}\right) \in \bar{R}: x_{n}=c_{n}+\frac{1}{2} \ell_{n}(R)\right\}$, and analogously is defined the lower face $F_{R}^{l}$. Given a rectangle $R$, we denote $a R(a>1)$, the expanded rectangle centered in $c_{R}$ with edges $\ell_{i}(a R)=a \ell_{i}(R)$. With $z_{R}$ we denote the last coordinate of points belonging to $F_{R}^{l}$, that is $z_{R}=c_{n}-\frac{1}{2} \ell_{n}(R)$. We say that $R_{1}$ and $R_{2}$ are touching rectangles if $R_{1} \cap R_{2}=\emptyset$ and $\bar{R}_{1} \cap \bar{R}_{2}=F$ with $F$ a face of $R_{1}$ or $R_{2}$.

When we consider external cusps, with tip at the origin, we write, for every $x \in \mathbb{R}^{n}, d=d(x)$ for the distance to the origin. For any $p, 1<p<\infty, p^{\prime}$ stands for the conjugate exponent of $p$, $\frac{1}{p}+\frac{1}{p^{\prime}}=1$. For a matrix $A \in \mathbb{R}^{n \times n}$ we denote: $|A|^{p}=\sum_{i=1}^{n} \sum_{j=1}^{n}\left|A_{i, j}\right|^{p}$, and analogoulsy for vectors $v \in \mathbb{R}^{n}$.

Let $\omega: \Omega \longrightarrow \mathbb{R}_{\geq 0}$ be, a measurable function, the weighted $L_{\omega}^{p}$ norm of a matrix field $A: \mathbb{R}^{n} \longrightarrow \mathbb{R}^{n \times n}$ is defined as

$$
\|A\|_{L_{\omega}^{p}(\Omega)^{n \times n}}^{p}:=\int_{\Omega}|A|^{p} \omega=\sum_{i=1}^{n} \sum_{j=1}^{n}\left\|\omega^{\frac{1}{p}} A_{i, j}\right\|_{L^{p}(\Omega)}^{p},
$$

and analogously for vector fields. The weighted Sobolev space $W_{\omega}^{1, p}(\Omega)^{n}$ is defined by the set of vector fields $u: \Omega \rightarrow \mathbb{R}^{n}$ for which

$$
\|u\|_{W_{\omega}^{1, p}(\Omega)^{n}}^{p}:=\sum_{i=1}^{n} \int_{\Omega}\left(|u|^{p}+|D u(x)|^{p}\right) \omega=\sum_{i=1}^{n} \sum_{|\alpha| \leq 1}\left\|\omega^{\frac{1}{p}} D^{\alpha} u_{i}\right\|_{L^{p}(\Omega)}^{p},
$$

is finite. In the case $\omega \equiv 1$, we drop the subscript and write $\|u\|_{W^{1, p}(\Omega)^{n}}$ and $\|A\|_{W^{1, p}(\Omega)^{n \times n}}$, respectively. The average value of a function $u$ over a domain $D$ is written with either of the following notations: $u_{D}:=f_{D} u:=\frac{1}{|D|} \int_{D} u$, where $|D|$ stands for the measure of $D$, and analogously for the weighted average we write $u_{D_{\omega}}:=f_{D_{\omega}} u:=\frac{1}{\omega(D)} \int_{D} u \omega$

Let us state the following discrete weighted inequality of Hardy type [21, page 52]:

Lemma 2.1. Let $\left\{u_{i}\right\}_{i}$ and $\left\{v_{i}\right\}_{i}$ be sequences of non-negative weights; and let $1<p \leq q<\infty$. Then the inequality:

$$
\left[\sum_{j=1}^{\infty} u_{j}\left(\sum_{i=1}^{j} b_{i}\right)^{q}\right]^{\frac{1}{q}} \leq c\left[\sum_{j=1}^{\infty} v_{j} b_{j}^{p}\right]^{\frac{1}{p}}
$$

holds for every non-negative sequences $\left\{b_{i}\right\}_{i}$ if

$$
\mathbf{A}=\sup _{k>0}\left(\sum_{j=k}^{\infty} u_{j}\right)^{\frac{1}{q}}\left(\sum_{j=0}^{k} v_{j}^{1-p^{\prime}}\right)^{\frac{1}{p^{\prime}}}<\infty
$$

The constant $c$ is $c=M \mathbf{A}$, where $M$ depends only on $p$ and $q$.

Throughout this paper we make extensive use of the following Lemma.

Lemma 2.2. Let $\left\{r_{i}\right\}_{i}$ and $\mathbf{a}=\left\{a_{i}\right\}_{i}$ be sequences such that $\left\{r_{i}\right\}_{i}>0$, and $\sum_{i} r_{i}=r<\infty$. Let us denote

$$
\bar{a}=\frac{1}{r} \sum_{j} a_{j} r_{j} .
$$

Then the inequality:

$$
\left(\sum_{j=1}^{\infty}\left|a_{j}-\bar{a}\right|^{p} r_{j}\right)^{\frac{1}{p}} \leq c\left(\sum_{j=1}^{\infty}\left|a_{j+1}-a_{j}\right|^{p} r_{j+1}\right)^{\frac{1}{p}}
$$


holds if

$$
\mathbf{A}=\sup _{k>0}\left(\sum_{j=k}^{\infty} r_{j}\right)^{\frac{1}{p}}\left(\sum_{j=0}^{k} r_{j}^{1-p^{\prime}}\right)^{\frac{1}{p^{\prime}}}<\infty
$$

The constant $c$ is $c=M \mathbf{A}$ where $M$ depends only on $p$.

Proof. Let us define the norm:

$$
\|\mathbf{a}\|_{p}=\left(\sum_{i}\left|a_{i}\right|^{p} r_{i}\right)^{\frac{1}{p}}
$$

From Hölder's inequality, it holds $|\bar{a}| r \leq\|\mathbf{a}\|_{p} r^{\frac{1}{p^{\prime}}}$ and then $\|\mathbf{a}-\bar{a}\|_{p} \leq 2\|\mathbf{a}\|_{p}$. Applying this last inequality with a replaced by $\mathbf{a}-a_{0}$, we obtain

$$
\|\mathbf{a}-\bar{a}\|_{p} \leq 2\left\|\mathbf{a}-a_{0}\right\|_{p} .
$$

Therefore:

$$
\sum_{i}\left|a_{i}-\bar{a}\right|^{p} r_{i} \leq 2^{p} \sum_{i}\left|a_{i}-a_{0}\right|^{p} r_{i} \leq 2^{p} \sum_{i}\left(\sum_{j=1}^{i}\left|a_{j}-a_{j-1}\right|\right)^{p} r_{i}
$$

And we conclude applying Lemma 2.1 with $u_{i}=v_{i}=r_{i}, q=p$ and $b_{i}=\left|a_{i}-a_{i-1}\right|$.

Remark 2.3. Observe that if in Lemma 2.D, $\left\{r_{i}\right\}_{1 \leq i \leq N}$ is finite and $r_{i} \underset{C}{\sim} r$ for any $i$, then

$$
\mathbf{A}=\max _{N \geq k>0}\left(\sum_{j=k}^{N} r_{j}\right)^{\frac{1}{p}}\left(\sum_{j=0}^{k} r_{j}^{1-p^{\prime}}\right)^{\frac{1}{p^{\prime}}} \leq C N
$$

Proof. In fact,

$$
\begin{aligned}
\mathbf{A} & =\max _{N \geq k>0}\left(\sum_{j=k}^{N} r_{j}\right)^{\frac{1}{p}}\left(\sum_{j=0}^{k} r_{j}^{1-p^{\prime}}\right)^{\frac{1}{p^{\prime}}} \leq C \max _{N \geq k>0}\left(\sum_{j=k}^{N} r\right)^{\frac{1}{p}}\left(\sum_{j=0}^{k} r^{1-p^{\prime}}\right)^{\frac{1}{p^{\prime}}} \\
& =C \max _{N \geq k>0}((N-k) r)^{\frac{1}{p}}\left(k r^{1-p^{\prime}}\right)^{\frac{1}{p^{\prime}}}=\max _{N \geq k>0}(N-k)^{\frac{1}{p}} k^{\frac{1}{p^{\prime}}} r^{\frac{1}{p}+\frac{1}{p^{\prime}}-1} \leq C N
\end{aligned}
$$

\section{Korn and Poincaré Inequalities for Chains of Rectangles}

Definition 3.1. A (finite or countable) collection of rectangles $\mathcal{C}=\left\{R_{i}\right\}$ for which $\sum_{i}\left|R_{i}\right|<\infty$, is called a chain of rectangles if a) $\bar{R}_{i} \cap \bar{R}_{j}=\emptyset$ for $|i-j|>1$, b) for any $i, R_{i}$ and $R_{i+1}$ are touching, and c) there exists a constant $C$ such that $R_{i} \underset{C}{\sim} R_{i+1}$, for any $i$.

Remark 3.1. Given a chain of rectangles $\mathcal{C}=\left\{R_{i}\right\}$ we have the following elementary facts

- since $R_{i}$ and $R_{i+1}$ are touching and $C$-comparable there exists a rectangle $R_{i, i+1} \subset$ $\bar{R}_{i} \cup \bar{R}_{i+1}$ and a constant $\tilde{C}$ depending only on $C$, such that

$$
R_{i, i+1} \underset{\tilde{C}}{\sim}\left(R_{i, i+1} \cap R_{i}\right) \underset{\tilde{C}}{\sim} R_{i} \underset{\tilde{C}}{\sim}\left(R_{i, i+1} \cap R_{i+1}\right) \underset{\tilde{C}}{\sim} R_{i+1}
$$

- thanks to the previous item

$$
\left|R_{i, i+1}\right| \underset{\tilde{C}}{\sim}\left|\left(R_{i, i+1} \cap R_{i}\right)\right| \underset{\tilde{C}}{\sim}\left|R_{i}\right| \underset{\tilde{C}}{\sim}\left|\left(R_{i, i+1} \cap R_{i+1}\right)\right| \underset{\tilde{C}}{\sim}\left|R_{i+1}\right|
$$

with $\tilde{C}$ depending only on $C$. 
- using that $R_{i}, R_{i+1}$ and $R_{i, i+1}$ are $\tilde{C}$-comparable we get (for instance by changing variables) that there exists a constant $\hat{C}$, depending only on $\tilde{C}$ (therefore only on $C$ ) such that

$$
C_{i} \underset{\hat{C}}{\sim} C_{i+1} \underset{\hat{C}}{\sim} C_{i, i+1}
$$

being $C_{i}, C_{i+1}, C_{i, i+1}$ the constants in the second case of Korn's inequality for $R_{i}, R_{i+1}$ and $R_{i, i+1}$ respectively.

Definition 3.2. Given a chain of rectangles $\mathcal{C}$, any given collection of intermediate rectangles $R_{i, i+1}$ enjoying properties like those mentioned in Remark 3.1 is denoted with $\mathcal{C}_{I}=\left\{R_{i, i+1}\right\}$.

Definition 3.3. Given a chain of rectangles $\mathcal{C}$, we call $\Omega=\cup\left(\mathcal{C}_{I} \cup \mathcal{C}\right)$ a $\mathcal{C}$-linked domain.

Remark 3.2. Observe that if $\Omega_{1} \subset \Omega_{2}$ and $\left|\Omega_{2} \backslash \Omega_{1}\right|=0$, then $K_{\Omega_{1}} \geq K_{\Omega_{2}}$ and $P_{\Omega_{1}} \geq P_{\Omega_{2}}$, where $K_{\Omega_{j}}$ and $P_{\Omega_{j}}$ are the constants for the second case of Korn's inequality and of Poincaré's inequality on $\Omega_{j}$, respectively. Consequently, the results that we prove for $\mathcal{C}-$ linked domains hold for every $\Omega$ such that $\cup\left(\mathcal{C}_{i} \cup \mathcal{C}\right) \subset \Omega$ and $\Omega \equiv \cup\left(\mathcal{C}_{I} \cup \mathcal{C}\right)$.

Theorem 3.3 (Second Case of Korn's Inequality for Chains of Rectangles). Let $\mathcal{C}=\left\{R_{i}\right\}$ be a chain of rectangles, and let $C_{i}$ be the constants for the second case of Korn's inequality on $R_{i}$. Then for any $\mathcal{C}$ - linked domain $\Omega$, and any $u \in W^{1, p}(\Omega)^{n}$ such that $f_{\Omega} \frac{D u-D u^{t}}{2}=0$ we have

$$
\|D u\|_{L^{p}(\Omega)^{n \times n}} \leq C(1+\mathbf{A})\|\varepsilon(u)\|_{L_{\sigma}^{p}(R)^{n \times n}},
$$

where $\mathbf{A}$ is defined in (2.2) with $r_{j}=\left|R_{j}\right|$, and the weight $\sigma$ is constant on each $R_{i}$ being $\left.\sigma\right|_{R_{i}}=C_{i}^{p}$.

Proof. Let

$$
A^{i}=\frac{1}{2\left|R_{i}\right|} \int_{R_{i}}\left(D u-D u^{t}\right)
$$

Then:

$$
\|D u\|_{L^{p}(\Omega)^{n \times n}}^{p}=\sum_{i}\|D u\|_{L^{p}\left(R_{i}\right)^{n \times n}}^{p} \leq \underbrace{C \sum_{i}\left\|D u-A^{i}\right\|_{L^{p}\left(R_{i}\right)^{n \times n}}^{p}}_{I}+\underbrace{C \sum_{i}\left\|A^{i}\right\|_{L^{p}\left(R_{i}\right)^{n \times n}}^{p}}_{I I}
$$

$I$ leads to

$$
I \leq C \sum_{i} C_{i}^{p}\|\varepsilon(u)\|_{L^{p}\left(R_{i}\right)^{n \times n}}^{p} \leq C \sum_{i}\|\varepsilon(u)\|_{L_{\sigma}^{p}\left(R_{i}\right)^{n \times n}}^{p}=C\|\varepsilon(u)\|_{L_{\sigma}^{p}(\Omega)^{n \times n}}^{p}
$$

For $I I$, apply inequality (2.1) with $r_{j}=\left|R_{j}\right|$. Let us observe that $\sum\left|R_{i}\right| A^{i}=0$, therefore taking

$$
\mathbf{A}=\sup _{k>0}\left(\sum_{j \geq k}\left|R_{j}\right|\right)^{\frac{1}{p}}\left(\sum_{j \leq k}\left|R_{j}\right|^{1-p^{\prime}}\right)^{\frac{1}{p^{\prime}}}
$$

we have

$$
I I=C \sum_{i}\left|A^{i}\right|^{p}\left|R_{i}\right| \leq C \mathbf{A}^{p} \sum_{i}\left|A^{i+1}-A^{i}\right|^{p}\left|R_{i+1}\right|
$$

where $C$ is a constant depending on $n$ and $p$. For each $i$, let us now introduce a rectangle $R_{i, i+1}$ as defined in Remark 3.1. Calling

$$
A^{i, i+1}=\frac{1}{2\left|R_{i, i+1}\right|} \int_{R_{i, i+1}} D u-D u^{t}
$$


we get, using extensively Remark 3.1,

$$
\begin{aligned}
I I & \leq C \mathbf{A}^{p} \sum_{i}\left\{\left|A^{i+1}-A^{i, i+1}\right|^{p}+\left|A^{i, i+1}-A^{i}\right|^{p}\right\}\left|R_{i+1}\right| \\
& \leq C \mathbf{A}^{p} \sum_{i}\left\{\left|A^{i+1}-A^{i, i+1}\right|^{p}\left|R_{i+1} \cap R_{i, i+1}\right|+\left|A^{i, i+1}-A^{i}\right|^{p}\left|R_{i} \cap R_{i, i+1}\right|\right\} \\
& =C \mathbf{A}^{p} \sum_{i}\left\{\left\|A^{i+1}-A^{i, i+1}\right\|_{L^{p}\left(R_{i+1} \cap R_{i, i+1}\right)}^{p}+\left\|A^{i}-A^{i, i+1}\right\|_{L^{p}\left(R_{i} \cap R_{i, i+1}\right)}^{p}\right\} \\
& \leq C \mathbf{A}^{p} \sum_{i}\left\{\left\|A^{i+1}-D u\right\|_{L^{p}\left(R_{i+1}\right)^{n \times n}}^{p}+\left\|D u-A^{i, i+1}\right\|_{L^{p}\left(R_{i, i+1}\right)^{n \times n}}^{p}+\left\|D u-A^{i}\right\|_{L^{p}\left(R_{i}\right)^{n \times n}}^{p}\right\} \\
& \leq C \mathbf{A}^{p} \sum_{i} C_{i}^{p}\|\varepsilon(u)\|_{L^{p}\left(R_{i+1} \cup R_{i}\right)^{n \times n}}^{p} \\
& \leq C \mathbf{A}^{p} \sum_{i} C_{i}^{p}\|\varepsilon(u)\|_{L^{p}\left(R_{i}^{n \times n}\right)}^{p}
\end{aligned}
$$

where, in the last inequality we use that for each $R_{i}, \bar{R}_{i} \cap \bar{R}_{j}=\emptyset$ if $|i-j|>1$. Therefore

$$
I I \leq C \mathbf{A}^{p}\|\varepsilon(u)\|_{L_{\sigma}^{p}(\Omega)^{n \times n}}^{p}
$$

and the Theorem follows.

Remark 3.4. In a recent paper, $R$. Durán proves that the constant for the second case of Korn's inequality for any convex domain $\Omega$ can be bounded taking the quotient between the diameter of $\Omega$ and the diameter of a maximal ball contained in $\Omega$ (see [12, Theorem 4.2]). Even when in [12] that result is stated only for $p=2$, the same proof works for $1<p<\infty$. It is important to notice that this implies that given a rectangle $R$ with edges $\ell_{i}(R)$, eventually all different, Korn's constant in the second case can be taken $\frac{L_{M}\left(R_{i}\right)}{L_{m}\left(R_{i}\right)}$. That estimate is sharp. Take, for instance, $n=2$ and $u(x, y)=\left(-x y, \frac{x^{2}}{2}\right)$, defined over $R=\left(0, L_{M}\right) \times\left(-\frac{L_{m}}{2}, \frac{L_{m}}{2}\right)$ we have that

$$
\|D u\|_{L^{p}(R)^{n \times n}}^{p} \underset{C}{\sim} L_{m} L_{M}^{p+1} \quad \text { and } \quad\|\varepsilon(u)\|_{L^{p}(R)^{n \times n}}^{p} \underset{C}{\sim} L_{m}^{p+1} L_{M},
$$

and therefore

$$
\frac{\|D u\|_{L^{p}(R)^{n \times n}}^{p}}{\|\varepsilon(u)\|_{L^{p}(R)^{n \times n}}^{p}} \widetilde{C}\left(\frac{L_{M}}{L_{m}}\right)^{p} .
$$

Remark 3.5. Thanks to the previous Remark, in Theorem 3.3, $C_{i}$ can be taken as follows

$$
C_{i}=\frac{L_{M_{i}}}{L_{m_{i}}}
$$

Theorem 3.3 can be straightforwardly extended to some weighted spaces.

Definition 3.4. Let $\mathcal{C}=\left\{R_{i}\right\}$, be a chain of rectangles, and $\Omega$ a domain such that $\Omega \equiv \cup \mathcal{C}$. We say that $\omega$ is an admissible weight in $\Omega$ if for any $x \in R_{i}$

$$
\omega(x) \underset{C}{\sim} \omega_{R_{i}} \underset{C}{\sim} \omega_{R_{i+1}} \forall i .
$$

being $\omega_{R_{i}}$ appropriate constants.

aa

The following is an elementary generalization of Theorem 3.3

\footnotetext{
${ }^{1}$ Observe that aligning $N$ identical cubes in a rectangle $R$ we have that Remark 2.3 yields such a constant, written there in terms of $N=L_{M}(R) / L_{m}(R)$.
} 
Theorem 3.6 (Second Case of Weighted Korn's Inequality for Chains of Rectangles). Let $\mathcal{C}=\left\{R_{i}\right\}$ be a chain of rectangles and $\Omega$ a $\mathcal{C}$ - linked domain.

Let $u \in W_{\omega}^{1, p}(\Omega)^{n}$, with $\omega$ an admissible weight (see (3.2)), be such that

$$
\int_{\Omega} \frac{D u-D u^{t}}{2} \omega=0
$$

If $\sum_{i} \omega\left(R_{i}\right)=r<\infty$,

$$
\|D u\|_{L_{\omega}^{p}(\Omega)^{n \times n}} \leq C\left(1+\mathbf{A}_{\omega}\right)\|\varepsilon\|_{L_{\omega \sigma}^{p}(\Omega)^{n \times n}}
$$

where $\left.\sigma\right|_{R_{i}}$ can be taken as in Theorem 3.3, and

$$
\mathbf{A}_{\omega}:=\sup _{k>0}\left(\sum_{j \geq k} \omega\left(R_{j}\right)\right)^{\frac{1}{p}}\left(\sum_{j \leq k} \omega\left(R_{j}\right)^{1-p^{\prime}}\right)^{\frac{1}{p^{\prime}}}
$$

Proof. Let:

$$
A^{i}=\frac{1}{\left|R_{i}\right|} \int_{R_{i}} \frac{D u-D u^{t}}{2} \quad \text { and } \quad A_{\omega}^{i}=\frac{1}{\omega\left(R_{i}\right)} \int_{R_{i}} \frac{D u-D u^{t}}{2} \omega
$$

We take:

$$
\|D u\|_{L_{\omega}^{p}(\Omega)^{n \times n}}^{p}=\sum_{i}\|D u\|_{L_{\omega}^{p}\left(R_{i}\right)^{n \times n}}^{p} \leq C\{\underbrace{\sum_{i}\left\|D u-A_{\omega}^{i}\right\|_{L_{\omega}^{p}\left(R_{i}\right)^{n \times n}}^{p}}_{(a)}+\underbrace{\left.\sum_{i}\left\|A_{\omega}^{i}\right\|_{L_{\omega}^{p}\left(R_{i}\right)^{n \times n}}^{p}\right\}}_{(b)}
$$

For $(a)$ we write

$$
\left\|D u-A_{\omega}^{i}\right\|_{L_{\omega}^{p}\left(R_{i}\right)^{n \times n}} \leq \underbrace{\left\|D u-A^{i}\right\|_{L_{\omega}^{p}\left(R_{i}\right)^{n \times n}}}_{I}+\underbrace{\left\|A^{i}-A_{\omega}^{i}\right\|_{L_{\omega}^{p}\left(R_{i}\right)^{n \times n}}}_{I I}
$$

and for I, we can take the weight off the norms

$$
\begin{aligned}
I^{p} & =\left\|D u-A^{i}\right\|_{L_{\omega}^{p}\left(R_{i}\right)^{n \times n}}^{p} \leq \omega_{R_{i}}^{p}\left\|D u-A^{i}\right\|_{L^{p}\left(R_{i}\right)^{n \times n}}^{p} \leq C \omega_{R_{i}}^{p}\|\varepsilon(u)\|_{L_{\sigma}^{p}\left(R_{i}\right)}^{p} \\
& \leq C\|\varepsilon(u)\|_{L_{\omega \sigma}^{p}\left(R_{i}\right)}^{p}
\end{aligned}
$$

On the other hand

$$
\begin{aligned}
I I^{p} & =\left\|A^{i}-A_{\omega}^{i}\right\|_{L_{\omega}^{p}\left(R_{i}\right)^{n \times n}}^{p}=\omega\left(R_{i}\right)\left|A^{i}-\frac{1}{\omega\left(R_{i}\right)} \int_{R_{i}} \frac{D u-D u^{t}}{2} \omega(x) d x\right|^{p} \\
& =\omega\left(R_{i}\right)\left|\frac{1}{\omega\left(R_{i}\right)} \int_{R_{i}}\left(A^{i}-\frac{D u-D u^{t}}{2}\right) \omega(x) d x\right|^{p} \\
& \leq C \omega\left(R_{i}\right)^{1-p}\left\{\left|\int_{R_{i}}\left(A^{i}-D u\right) \omega(x) d x\right|^{p}+\left|\int_{R_{i}}\left(D u-\frac{D u-D u^{t}}{2}\right) \omega(x) d x\right|^{p}\right\} \\
& =C \omega\left(R_{i}\right)^{1-p}\left\{\left|\int_{R_{i}}\left(A^{i}-D u\right) \omega(x)^{\frac{1}{p}} \omega(x)^{\frac{1}{p^{p}}} d x\right|^{p}+\left|\int_{R_{i}} \varepsilon(u) \omega(x)^{\frac{1}{p}} \omega(x)^{\frac{1}{p^{\prime}}} d x\right|^{p}\right\}
\end{aligned}
$$

Applying Hölder inequality in both terms,

$$
\begin{aligned}
I I^{p} & \leq C \omega\left(R_{i}\right)^{1-p}\left\{\left\|A^{i}-D u\right\|_{L_{\omega}^{p}\left(R_{i}\right)^{n \times n}}^{p} \omega\left(R_{i}\right)^{\frac{p}{p^{\prime}}}+\|\varepsilon(u)\|_{L_{\omega}^{p}\left(R_{i}\right)^{n \times n}}^{p} \omega\left(R_{i}\right)^{\frac{p}{p^{\prime}}}\right\} \\
& =C\left\{I^{p}+\|\varepsilon(u)\|_{L_{\omega}^{p}\left(R_{i}\right)^{n \times n}}^{p}\right\} \leq C\|\varepsilon(u)\|_{L_{\omega \sigma}^{p}\left(R_{i}\right)}^{p}
\end{aligned}
$$


On the other hand, for $(b)$, let us observe that

$$
\sum_{i} \omega\left(R_{i}\right) A_{\omega}^{i}=0
$$

and that:

$$
\sum_{i}\left\|A_{\omega}^{i}\right\|_{L_{\omega}^{p}\left(R_{i}\right)^{n \times n}}^{p}=\sum_{i} \omega\left(R_{i}\right)\left|A_{\omega}^{i}\right|^{p}
$$

Consequently, Lemma 2.2 with $a_{i}=A_{\omega}^{i}$ and $r_{i}=\omega\left(R_{i}\right)$, yields

$$
\sum_{i}\left\|A_{\omega}^{i}\right\|_{L_{\omega}^{p}\left(R_{i}\right)^{n \times n}}^{p} \leq C \mathbf{A}_{\omega} \sum_{i=1}^{\infty}\left|A_{\omega}^{i+1}-A_{\omega}^{i}\right|^{p} \omega\left(R_{i+1}\right) \leq C \mathbf{A}_{\omega} \sum_{i=1}^{\infty}\left\|A_{\omega}^{i+1}-A_{\omega}^{i}\right\|_{L_{\omega}^{p}\left(R_{i+1}\right)}^{p},
$$

Now we may proceed like in Theorem 3.3 , alternating $A_{\omega}^{i, i+1}$, the weighted average of $(D u-$ $\left.D u^{t}\right) / 2$ on an overlaping rectangle $R_{i, i+1}$, afterwards alternating $D u$, and finally applying the estimates for $(a)$. We leave the final details to the reader.

Observe that Theorem 3.3 is a Corollary of the previous theorem taking $\omega \equiv 1$. However, Theorem 3.6 does not provide information unless $\mathbf{A}_{\omega}<\infty$. A simple way to bound $\mathbf{A}_{\omega}$ involves a reasonable decay for $\omega\left(R_{i}\right)$.

Corollary 3.7. Under the same hypotheses of Theorem 3.6. Assume that for any $k$,

$$
\omega\left(R_{k+1}\right) \leq \alpha \omega\left(R_{k}\right) \quad \text { with } 0 \leq \alpha<1 .
$$

Then for any $u \in W_{\omega}^{1, p}(\Omega)^{n}$ such that $\int_{\Omega} \frac{D u-D u^{t}}{2} \omega=0$, we have

$$
\|D u\|_{L_{\omega}^{p}(\Omega)^{n \times n}} \leq C\|\varepsilon(u)\|_{L_{\omega \sigma}^{p}(R)^{n \times n}}
$$

where the weight $\sigma$ is constant on each element of $\mathcal{C}$, and can be taken as $\left.\sigma\right|_{R_{i}}=\left(\frac{L_{M_{i}}}{L_{m_{i}}}\right)^{p}$.

Proof. From Remark 3.4, we know that $C_{i} \leq C \frac{L_{M_{i}}}{L_{m_{i}}}$ with $C_{i}$ defined in Theorem 3.3. Therefore only remains to show that $\sum_{i} \omega\left(R_{i}\right)<\infty$ and $\mathbf{A}_{\omega}<C$. These follow from the bounds $\omega\left(R_{k}\right) \leq$ $\alpha^{k-i} \omega\left(R_{i}\right)$ for $0 \leq i \leq k$ and $\omega\left(R_{i}\right) \leq \alpha^{i-k} \omega\left(R_{k}\right)$ for $i \geq k$. Indeed

$$
\mathbf{A}_{\omega}=\sup _{k>0}\left(\sum_{j=k}^{\infty} \omega\left(R_{j}\right)\right)^{\frac{1}{p}}\left(\sum_{j=0}^{k} \omega\left(R_{j}\right)^{1-p^{\prime}}\right)^{\frac{1}{p^{\prime}}} \leq \omega\left(R_{k}\right)^{\frac{1}{p}}\left(\sum_{j=0}^{\infty} \alpha^{j}\right)^{\frac{1}{p}} \omega\left(R_{k}\right)^{\frac{1}{p^{\prime}}-1}\left(\sum_{j=0}^{k} \alpha^{\left(p^{\prime}-1\right) j}\right)^{\frac{1}{p^{\prime}}}
$$

then

and the Corollary follows.

$$
\mathbf{A}_{\omega} \leq\left(\frac{1}{1-\alpha}\right)^{\frac{1}{p}}\left(\frac{1}{1-\alpha^{p^{\prime}-1}}\right)^{\frac{1}{p^{\prime}}}
$$

Everything done so far for the second case of Korn's inequality for chains of rectangles can be done for Poincaré inequality following step by step the arguments given above. Since the constant in Poincaré inequality for rectangles (and in general for convex domains) depends only on the diameter of the rectangle, the weight involved in the inequality can be weakened as it is stated below.

Theorem 3.8 (Poincaré inequality for Chains of Rectangles). Let $1<p<\infty$ and $\mathcal{C}=\left\{R_{i}\right\}$ be a chain of rectangles and $\Omega$ a $\mathcal{C}$-linked domain. Let $\omega$ be an addmissible weight (see (3.2)), such that for any $k, \omega\left(R_{k+1}\right) \leq \alpha \omega\left(R_{k}\right)$ with $0 \leq \alpha<1$. Then if $u \in W_{\omega}^{1, p}(\Omega)^{n}$, and $\int_{\Omega} u \omega=0$, we have

$$
\|u\|_{L_{\omega}^{p}(\Omega)} \leq C\|D u\|_{L_{\omega \sigma}^{p}(\Omega)^{n \times n}}
$$

where the weight $\sigma$ is constant on each $R_{i}$ and can be taken as $\left.\sigma\right|_{R_{i}}=L_{M_{i}}^{p}$. 
Remark 3.9. Let us mention that Theorem 3.8, as well as subsequent results involving Poincaré inequalities, also holds for $p=1$. The key point is that during the proof Lemma 2.2 is invoked with the choice $r_{i}=\omega\left(R_{i}\right)$ under the decay condition $\omega\left(R_{i+1}\right) \leq \alpha \omega\left(R_{i}\right)$. In this case it is easy to see, by a direct proof of Lemma 2.1 for $p=1$ taking $u_{i}=v_{i}=r_{i}$, that (2.1) holds for $p=1$ and $c=\frac{2}{1-\alpha}$.

The following version will be useful in the sequel.

Corollary 3.10. With the same hypotheses of Theorem 3.8, assume that $B$ is a ball such that $B \subset \Omega$, and $B \cap R_{j} \neq \emptyset$ only for a finite number of rectangles. Then, for every $u \in W_{\omega}^{1, p}(\Omega)^{n}$, we have:

$$
\|u\|_{L_{\omega}^{p}(\Omega)^{n}} \leq C\left\{\|u\|_{L^{p}(B)^{n}}+\|D u\|_{L_{\omega \sigma}^{p}(\Omega)^{n \times n}}\right\}
$$

where the weight $\sigma$ is constant on each $R_{i}$ and can be taken as $\left.\sigma\right|_{R_{i}}=L_{M_{i}}^{p}$.

Proof. For the sake of clarity we write the case $\omega \equiv 1$.

$$
\|u\|_{L^{p}(\Omega)^{n}} \leq\left\|u-u_{B}\right\|_{L^{p}(\Omega)^{n}}+\left\|u_{B}\right\|_{L^{p}(\Omega)^{n}} \leq \underbrace{\left\|u-u_{\Omega}\right\|_{L^{p}(\Omega)^{n}}}_{I}+\underbrace{\left\|u_{\Omega}-u_{B}\right\|_{L^{p}(\Omega)^{n}}}_{I I}+\underbrace{\left\|u_{B}\right\|_{L^{p}(\Omega)^{n}}}_{I I I} .
$$

Applying Theorem 3.8:

On the other hand,

$$
I \leq C\|D u\|_{L_{\sigma}^{p}(\Omega)^{n \times n}} .
$$

$$
I I I^{p}=\int_{\Omega}\left(f_{B} u\right)^{p}=\frac{|\Omega|}{|B|^{p}}\left(\int_{B} u\right)^{p} \leq \frac{|\Omega|}{|B|^{p}}|B|^{\frac{p}{p^{\prime}}} \int_{B} u^{p}=\frac{|\Omega|}{|B|}\|u\|_{L^{p}(B)^{n}}^{p} .
$$

For $I I$, applying Hölder inequality:

$$
\left|u_{\Omega}-u_{B}\right| \leq \frac{1}{|B|} \int_{B}\left|u_{\Omega}-u\right| \leq \frac{|B|^{\frac{1}{p^{p}}}}{|B|}\left\|u-u_{\Omega}\right\|_{L^{p}(B)} \leq \frac{1}{|B|^{\frac{1}{p}}}\left\|u-u_{\Omega}\right\|_{L^{p}(\Omega)^{n}} \leq \frac{C}{|B|^{\frac{1}{p}}}\|D u\|_{L^{p}(\Omega)_{\sigma}^{n \times n}}
$$

then

and the lemma follows for $\omega \equiv 1$.

$$
I I \leq C \frac{|\Omega|^{\frac{1}{p}}}{|B|^{\frac{1}{p}}}\|D u\|_{L^{p}(\Omega)_{\sigma}^{n \times n}},
$$

The general case follows similarly using (3.2), and taking into account that $B$ only meets a finite number of rectangles and then $\|u\|_{L_{\omega}^{p}(B)} \underset{C}{\sim}\|u\|_{L^{p}(B)}$.

We now prove the general case of Korn's inequality for chains of rectangles. Our proof is a straigthforward adaptation of the classic argument given in [3]. Let us notice that we require that $L_{M_{i}} \leq C$ for any $i$. That is in order to remove the weigth $\sigma$ from the Poincaré inequality given above.

Theorem 3.11 (General Case of Korn's inequality for Chains of Rectangles). Let $\mathcal{C}=\left\{R_{i}\right\}$ be a chain of rectangles, and $\Omega$ a $\mathcal{C}$ - linked domain. Consider a weight $\omega$ such that (3.2) holds, and assume that $\omega\left(R_{k+1}\right) \leq \alpha \omega\left(R_{k}\right)$ with $0 \leq \alpha<1$ and that $L_{M_{i}}<C$, for any $i$. If $B$ is a ball such that $B \subset \Omega$, and $B$ meets only a finite number of rectangles $R_{i}$ then for any $u \in W_{\omega}^{1, p}(\Omega)^{n}$, we have

$$
\|D u\|_{L_{\omega}^{p}(\Omega)^{n \times n}} \leq C\left\{\|u\|_{L^{p}(B)^{n}}+\|\varepsilon(u)\|_{L_{\omega \sigma}^{p}(\Omega)^{n \times n}}\right\},
$$

where the weight $\sigma$ is constant on each element of $\mathcal{C}$, and can be taken as $\left.\sigma\right|_{R_{i}}=\left(\frac{L_{M_{i}}}{L_{m_{i}}}\right)^{p}$. 
Proof. Again, let us focus first on the case $\omega \equiv 1$. Consider the space

$$
R M(\Omega)^{n}=\left\{v \in W^{1, p}(\Omega)^{n}: \quad \varepsilon(v)=0\right\},
$$

every function in $R M$ can be written as

$$
v(x)=a+M x,
$$

where $M \in \mathbb{R}^{n \times n}$ is skew symmetric. On the other hand, a complement of $R M$ in $W^{1, p}$ can be defined as follows

$$
\widehat{W}^{1, p}(\Omega)^{n}=\left\{w \in W^{1, p}(\Omega)^{n}: \quad f_{B} w=0, \quad f_{\Omega} \frac{D w-D w^{\prime}}{2}=0\right\} .
$$

In fact, given $u \in W^{1, p}(\Omega)^{n}$, we can take $v \in R M(\Omega)^{n}$ :

$$
v=a+M(x-\bar{x})
$$

with

$$
a=f_{B} u \quad \text { and } \quad m_{i j}=\frac{1}{2} f_{\Omega}\left(\frac{\partial u_{i}}{\partial x_{j}}-\frac{\partial u_{j}}{\partial x_{i}}\right)
$$

being $\bar{x}$ the center of $B$. Obviously $w=u-v \in \widehat{W}^{1, p}(\Omega)^{n}$, and in particular

$$
W^{1, p}(\Omega)^{n}=R M(\Omega)^{n} \oplus \widehat{W}^{1, p}(\Omega)^{n} .
$$

Moreover, it is clear by definition that

$$
\|v\|_{W^{1, p}(\Omega)^{n}} \leq C\|u\|_{W^{1, p}(\Omega)^{n}} \quad\|w\|_{W^{1, p}(\Omega)^{n}} \leq C\|u\|_{W^{1, p}(\Omega)^{n}} .
$$

If (3.5) does not hold, there is a sequence $\left\{u_{n}\right\} \subset W^{1, p}(\Omega)^{n}$ such that

$$
\left\|D u_{n}\right\|_{L^{p}(\Omega)^{n \times n}}=1
$$

but,

If we write

$$
\left\|u_{n}\right\|_{L^{p}(B)^{n}}+\left\|\varepsilon\left(u_{n}\right)\right\|_{L_{\sigma}^{p}(\Omega)^{n \times n}}<\frac{1}{n} .
$$

$$
u_{n}=v_{n}+w_{n}
$$

with $v_{n} \in R M(\Omega)^{n}$ and $w_{n} \in \widehat{W}^{1, p}(\Omega)^{n}, w_{n}$ admits both Poincaré inequality in $B$, and second case of Korn inequality in $\Omega$

$$
\begin{aligned}
\left\|w_{n}\right\|_{W^{1, p}(\Omega)^{n}} & =\left\|w_{n}\right\|_{L^{p}(\Omega)^{n}}+\left\|D w_{n}\right\|_{L^{p}(\Omega)^{n \times n}} \leq C\left(\left\|w_{n}\right\|_{L^{p}(B)^{n}}+\left\|D w_{n}\right\|_{L^{p}(g O)^{n \times n}}\right) \\
& \leq C\left\|D w_{n}\right\|_{L^{p}(\Omega)^{n \times n}} \leq C\left\|\varepsilon\left(w_{n}\right)\right\|_{L_{\sigma}^{p}\left(\Omega^{n \times n}\right)}<C \frac{1}{n}
\end{aligned}
$$

And then, $w_{n} \longrightarrow 0$ in $W^{1, p}$. On the other hand, $v_{n}$ belongs to the finite dimensional space $R M(\Omega)^{n}$ and is bounded on $\Omega$. Consequently, there is a subsequence, called again $v_{n}$, such that $v_{n} \longrightarrow v \in R M(\Omega)^{n}$ strongly in $W^{1, p}(B)^{n}$. As $w_{n} \longrightarrow 0$, we have that

$$
u_{n} \longrightarrow v \in R M(B)^{n} \quad \text { in } W^{1, p}(\Omega)^{n}
$$

But because of (3.7), $\|v\|_{L^{p}(B)^{n}}=0$, and $v$ is a linear function, so $v \equiv 0$ on $\Omega$, which contradicts (3.6), and the result follows in the case $\omega \equiv 1$. The general case can be treated by the same means defining the appropriate weighted versions $R M_{\omega}(\Omega)^{n}=\left\{v \in W_{\omega}^{1, p}(\Omega)^{n}: \quad \varepsilon(v)=0\right\}$, and $\widehat{W}_{\omega}^{1, p}(\Omega)^{n}=\left\{\widetilde{v} \in W_{\omega}^{1, p}(\Omega)^{n}: \quad \int_{B} \widetilde{v} \omega=0, \quad \int_{\Omega} \frac{D \widetilde{v}-D \widetilde{v}^{\prime}}{2} \omega=0\right\}$. 


\section{Korn and Poincaré Inequalities for Chains of Quasi-Rectangles}

The job done for chains of rectangles can be easily generalized to chains of more general sets, all we have to do is to write appropriate hypotheses.

Definition 4.1. Let $\mathcal{W}=\left\{\Omega_{i}\right\}$ be a (finite or countable) collection of disjoint open sets. Assume that there exists a chain of rectangles $\mathcal{C}=\left\{R_{i}\right\}$ (in the sense of Definition 3.1) with $R_{i} \subset$ $\Omega_{i} \subset C_{R} R_{i}$ for a fixed constant $C_{R}$. Finally assume that there exist fixed constants $C_{K}, C_{P}$ such that $C_{K_{i}} \leq C_{K} \frac{L_{M_{i}}}{L_{m_{i}}}$ and $C_{P_{i}} \leq C_{P} L_{M_{i}}$ being $C_{K_{i}}$ and $C_{P_{i}}$ the constants for the Korn's second inequality and Poincaré inequality respectively for $\Omega_{i}$. Then $\mathcal{W}=\left\{\Omega_{i}\right\}$ is called a $C$ chain of quasi-rectangles associated to the chain of rectangles $\mathcal{C}$. The constant $C$ is taken as $C=\max \left\{C_{R}, C_{K}, C_{P}\right\}$. Each $\Omega_{i}$ is called a $C$-quasi-rectangle associated to $R_{i}$.

In order to minimize the notation we use quasi-rectangle (resp. chain of quasi-rectangles) instead of $C$-quasi-rectangle (resp. C-chain of quasi-rectangles).

Definition 4.2. If each $R_{i}$ in Definition 4.1 is a cube $Q_{i}$ (instead of a general rectangle), then $\Omega_{i}$ is called a quasi-cube associated to the cube $Q_{i}$, and $C_{K_{i}} \leq C, C_{P_{i}} \leq C L_{Q_{i}}$.

Definition 4.3. Let $\mathcal{W}$ be a chain of quasi-rectangles associated to the chain of rectangles $\mathcal{C}$, and let $\mathcal{C}_{I}$ be a collection of intermediate rectangles associated to $\mathcal{C}$. We say that $\Omega=\cup\left(\mathcal{C}_{I} \cup \mathcal{W}\right)$ is a $\mathcal{W}$-linked domain.

Remark 4.1. Recalling Remark 3.2, the results that we state for $\mathcal{W}$-linked domains hold for every $\Omega$ such that $\cup\left(\mathcal{C}_{I} \cup \mathcal{W}\right) \subset \Omega$ and $\Omega \equiv \cup \mathcal{W}$.

Definition 4.4. Let $\mathcal{W}=\left\{\Omega_{i}\right\}$, be a chain of quasi-rectangles, and $\Omega$ a domain such that $\Omega \equiv \cup \mathcal{W}$. We say that $\omega$ is an admissible weight in $\Omega$ if for any $x \in \Omega_{i}$

$$
\omega(x) \underset{C}{\sim} \omega_{\Omega_{i}} \underset{C}{\sim} \omega_{\Omega_{i+1}} \forall i .
$$

being $\omega_{\Omega_{i}}$ appropriate constants.

Remark 4.2. From Definitions 4.1, 4.3 and 4.4 one readily finds that any proof given in previous section for $\mathcal{C}$ - linked domains can be carried out for $\mathcal{W}$ - linked domains. For this reason Theorems 4.3, 4.4, 4.5 and 4.6 are stated below without further analysis.

Theorem 4.3 (Second Case of Korn's Inequality for Chains of Quasi-Rectangles). Let $\mathcal{W}=\left\{\Omega_{i}\right\}$ be a chain of rectangles, and let $C_{K_{i}}$ be the constants for the second case of Korn's inequality on $\Omega_{i}$. Then for any $\mathcal{W}$ - linked domain $\Omega$, and any $u \in W^{1, p}(\Omega)^{n}$ such that $f_{\Omega} \frac{D u-D u^{t}}{2}=0$ we have

$$
\|D u\|_{L^{p}(\Omega)^{n \times n}} \leq C(1+\mathbf{A})\|\varepsilon(u)\|_{L_{\sigma}^{p}(\Omega)^{n \times n}},
$$

where $\mathbf{A}$ is defined in (2.2) with $r_{j}=\left|\Omega_{j}\right|$, and the weight $\sigma$ is constant on each $\Omega_{i}$ being $\left.\sigma\right|_{\Omega_{i}}=C_{K_{i}}^{p}$.

Theorem 4.4 (Second Case of Korn's Inequality for Chains of Quasi-Rectangles: weighted version). Let $\mathcal{W}=\left\{\Omega_{i}\right\}$ be a chain of quasi-rectangles and $\Omega$ a $\mathcal{W}$-linked domain. Assume that for any $k, \omega\left(R_{k+1}\right) \leq \alpha \omega\left(R_{k}\right)$ with $0 \leq \alpha<1$. Let $u \in W_{\omega}^{1, p}(\Omega)$, with $\omega$ an admissible weight (see (4.1)), be such that $\int_{\Omega} \frac{D u-D u^{t}}{2} \omega=0$. Then

$$
\|D u\|_{L_{\omega}^{p}(\Omega)^{n \times n}} \leq C\|\varepsilon\|_{L_{\omega \sigma}^{p}(\Omega)^{n \times n}}
$$

where $\left.\sigma\right|_{\Omega_{i}}$ can be taken as $\left.\sigma\right|_{R_{i}}=\left(\frac{L_{M_{i}}}{L_{m_{i}}}\right)^{p}$. 
Theorem 4.5 (Poincaré inequality for Chains of Rectangles). Let $1<p<\infty$ and $\mathcal{W}=\left\{\Omega_{i}\right\}$ be a chain of quasi rectangles and $\Omega$ a $\mathcal{W}$ - linked domain. Let $u \in W_{\omega}^{1, p}(\Omega)^{n}$, with $\omega$ an admissible weight (see (4.1)), be such that $u \in W_{\omega}^{1, p}(\Omega)^{n}$, and $\int_{\Omega} u \omega=0$. Assume that for any $k, \omega\left(R_{k+1}\right) \leq \alpha \omega\left(R_{k}\right)$ with $0 \leq \alpha<1$, then we have

$$
\|u\|_{L_{\omega}^{p}(\Omega)} \leq C\|D u\|_{L_{\omega \sigma}^{p}(\Omega)^{n \times n}},
$$

where the weight $\sigma$ is constant on each $\Omega_{i}$ and can be taken as $\left.\sigma\right|_{\Omega_{i}}=L_{M_{i}}^{p}$.

Theorem 4.6 (General Case of Korn's inequality for Chains of Quasi-Rectangles). Let $\mathcal{W}=$ $\left\{\Omega_{i}\right\}$ be a chain of rectangles, and $\Omega$ a $\mathcal{W}$-linked domain. Consider a weight $\omega$ such that (4.1) holds, and assume that $\omega\left(R_{k+1}\right) \leq \alpha \omega\left(R_{k}\right)$ with $0 \leq \alpha<1$ and that $L_{M_{i}}<C$, for any $i$. If $B$ is a ball such that $B \subset \Omega$, and $B$ meets only a finite number of quasi-rectangles $\Omega_{i}$ then for any $u \in W_{\omega}^{1, p}(\Omega)^{n}$, we have

$$
\|D u\|_{L_{\omega}^{p}(\Omega)^{n \times n}} \leq C\left\{\|u\|_{L^{p}(B)^{n}}+\|\varepsilon(u)\|_{L_{\omega \sigma}^{p}(\Omega)^{n \times n}}\right\},
$$

where the weight $\sigma$ is constant on each element of $\mathcal{C}$, and can be taken as $\left.\sigma\right|_{R_{i}}=\left(\frac{L_{M_{i}}}{L_{m_{i}}}\right)^{p}$.

Remark 4.7. All these results can be proved exactly like the ones for chains of rectangles, except for a subtle detail: we impose the decreasing measure condition (3.4) on the rectangles $R_{i}$ and not on the subdomains $\Omega_{i}$, as it would be natural. This is possible because of the relationship between the measures of $\Omega_{i}$ and $R_{i}$. Indeed, since $\omega$ is admissible and $\left|R_{i}\right| \leq\left|\Omega_{i}\right| \leq C\left|R_{i}\right|$, we have

$$
\omega\left(\Omega_{i}\right) \leq C \omega_{i}\left|\Omega_{i}\right| \leq C \omega_{i}\left|R_{i}\right| \leq C \omega\left(R_{i}\right)
$$

and

$$
\omega\left(R_{i}\right) \leq C \omega_{i}\left|R_{i}\right| \leq C \omega_{i}\left|\Omega_{i}\right| \leq C \omega\left(\Omega_{i}\right)
$$

And consequently:

$$
\mathbf{A}_{\omega}=\sup _{k>0}\left(\sum_{j=k}^{\infty} \omega\left(\Omega_{j}\right)\right)^{\frac{1}{p}}\left(\sum_{j=0}^{k} \omega\left(\Omega_{j}\right)^{1-p^{\prime}}\right)^{\frac{1}{p^{\prime}}} \leq C \sup _{k>0}\left(\sum_{j=k}^{\infty} \omega\left(R_{j}\right)\right)^{\frac{1}{p}}\left(\sum_{j=0}^{k} \omega\left(R_{j}\right)^{1-p^{\prime}}\right)^{\frac{1}{p^{\prime}}} .
$$

So, if the decreasing property (3.4) is imposed on the rectangles $R_{i}$ we have that $\mathbf{A}_{\omega}$ is finite.

The reader may wonder how a quasi-rectagle could be. An easy corollary of Theorem 4.3, useful in the next section, shows that some quasi-rectangles can be obtained from finite union of quasi-cubes.

Corollary 4.8. Let $\mathcal{W}=\left\{\Omega_{i}\right\}_{1 \leq i \leq N}$ be a a finite chain of quasi-cubes associated to a finite chain of cubes $\mathcal{C}=\left\{Q_{i}\right\}_{1 \leq i \leq N}$ with their centers placed along a straight line parallel to an axis. Assume that for $1 \leq i \leq N, \ell\left(Q_{i}\right)=\ell$. Then any $\mathcal{W}$ - linked domain $\Omega$ is a quasi-rectangle associated to $R$, being $R$ the minimal rectangle containing the chain $\mathcal{C}$.

Proof. It is enough to show that Korn and Poincaré constants $C_{K}$ and $C_{P}$ can be bounded as

$$
C_{K} \leq C \frac{L_{M}(R)}{L_{m}(R)}
$$

and

$$
C_{P} \leq C L_{M}(R)
$$

respectively. We show how to handle (4.3) since the other one follows similarly. We use Theorem 4.3 applied to chain $\mathcal{W}$. Since $\left|\Omega_{i}\right| \underset{C}{\widetilde{N}}\left|Q_{i}\right|=\ell^{n}$, we get from (2.2) and Remark 2.3 that $\mathbf{A} \leq C N$. 
On the other hand $N=\frac{L_{M}(R)}{L_{m}(R)}$, and then (4.3) follows straightforwardly, since by definition of quasi-cube $C_{K}\left(\Omega_{i}\right) \leq C \frac{L_{M}\left(Q_{i}\right)}{L_{m}\left(Q_{i}\right)}=C$.

Observe that this corollary only provides quasi-rectangles with interior rectangles having $n-1$ equal short edges and a long one. We limit our approach to that kind of quasi-rectangles in the context of external cusps treated later.

Taking into account that we work with quasi-rectangles that are made of quasi-cubes we need now to provide examples of general $C$-quasi-cubes.

A non trivial quasi-cube is given, for instance, by any bounded star-shaped domain with respect to a maximal ball $B$ contained in a cube $Q$. For such a domain $D$, calling $C_{Q}$ a constant for which $Q \subset D \subset C_{Q} Q$, it is known that second case of Korn and Poincaré inequalities hold. For star-shaped domains, some information about the constants $D_{K}$ and $D_{P}$ for Korn's and Poincaré inequality respectively is available in the literature (see [12], [13] [22]). However, all we need, in order to build quasi-rectangles, is to find a chain of quasi-cubes $\mathcal{W}=\left\{D_{i}\right\}$ for which $C_{K_{i}} \leq C$ and $C_{P_{i}} \leq C \ell(Q)$. This behavior of the constants is guaranteed in general for John domains, thanks to the solvability of the divergence equation [11] and the validity of the (improved) Poincaré inequality [23].

In Figure 2a we show a John domain which is a quasi-cube obtained by adding iteratively properly scaled cubes to a central fixed cube. In Figure $2 \mathrm{~b}$ a quasi-rectangle is given by collecting identical quasi-cubes like the one in Figure 2a. Finally Figure 2c shows a quasi-rectangle formed by a finite union of quasi-cubes that are not identical, but have similar aspect ratio.

Preceding remarks lead to the following example.

Example 4.1 (Locally John quasi-rectangle). Let $\mathcal{C}=\left\{Q_{i}\right\}, i=1, \ldots, N$ a chain of cubes with $\ell\left(Q_{i}\right)=\ell$ and with centers $c_{Q_{i}}$ placed along a straight line. Let $\mathcal{U}=\left\{\Omega_{i}\right\}, i=1, \ldots, N$ a set of disjoint John domains with parameters $\alpha, \beta$ and with centers in $c_{Q_{i}}$ such that $Q_{i} \subset \Omega_{i} \subset C_{i} Q_{i}$. Then any $\mathcal{U}$-linked domain $\Omega$ is a quasi-rectangle associated to $R$, being $R$ the minimal rectangle containing $\cup \mathcal{C}$. This sort of quasi-rectangle is called a locally John quasi-rectangle.

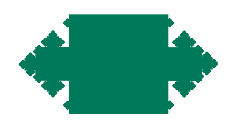

(A) Non-trivial quasi-cube

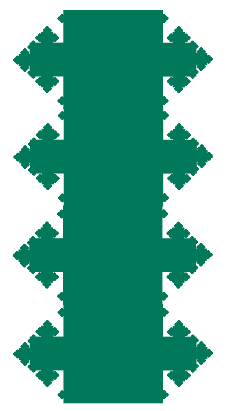

(B) Non-trivial quasi-rectangle

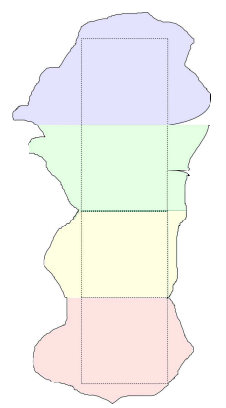

(c) Another quasirectangle

FiguRE 2. Quasi-rectangles

\section{Application to External Cusps}

External cusps are easily described using a "profile" function $\varphi$, as presented in (1.10). In particular if $\varphi\left(x_{n}\right) \underset{C}{\widetilde{x}} x_{n}^{\gamma}$ the weighted version of Korn's inequality given in Theorem A was presented in [15, Theorem 6.2]. A more general class of external cusps can be defined as follows: 
Definition 5.1. Let $\varpi \subset R^{n-1}$ a Lipschitz domain, assume that $0 \in \varpi$. We say that $\Omega$ is a sectionally Lipschitz external cusp if

$$
\Omega \cap U=\left\{\left(x^{\prime}, x_{n}\right) \in \mathbb{R}^{n-1} \times \mathbb{R}: x^{\prime} \in \varphi\left(x_{n}\right) \varpi\right\}
$$

for some neighborhood of the origin $U$, and with $\varphi$ a non-dereasing $C^{1}$ function, such that $\varphi$ in non-decreasing, $\varphi(0)=0$ and $\varphi(t) / t \rightarrow 0\left(t \rightarrow 0^{+}\right)$.

This definition is introduced in [24], in the context of extension of functions in Sobolev spaces. In fact, in [24] the authors drop the requirement $0 \in \varpi$ that here is considered for the sake of simplicity. In Figure 3, we show two external cusps satisfying Maz'ya and Poborchiî's definition. In Figure $3 \mathrm{a}, \varpi$ is an ellipse containing the origin , whereas in Figure $3 \mathrm{~b}, \varpi$ is an ellipse that does not contain the origin.

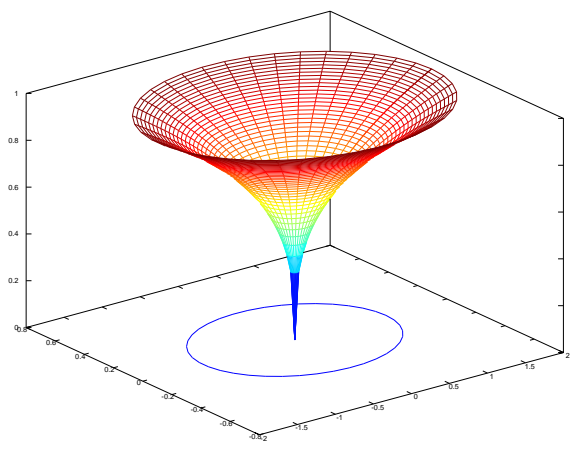

(A) Cusp containing the vertical axis

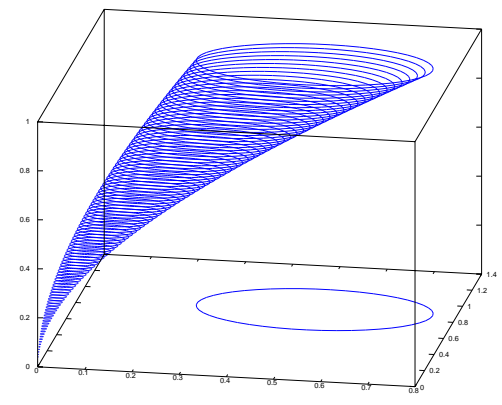

(в) Cusp tangential to the vertical axis

Figure 3. Maz'ya and Poborchiı̌'s cusps.

In this work we consider external cusps of a very general kind given by linking appropriate chains of quasi-rectangles.

Definition 5.2 (Generalized External Cusp). Let $\mathcal{W}=\left\{\Omega_{i}\right\}$ be a chain of quasi-rectangles with an associated chain of rectangles $\mathcal{C}=\left\{R_{i}\right\}$. Let us assume that:

- each $R_{i}$ is such that $\vec{\ell}\left(R_{i}\right)=\left(\ell_{i}, \ell_{i}, \cdots, \ell_{i}, L_{i}\right)$, with $\ell_{i} \leq L_{i}$.

- the rectangles $R_{i}$ are placed one above the other, along the $x_{n}$ axis in such a way that $\bar{R}_{i+1} \cap \bar{R}_{i}=F_{R_{i+1}}^{u}$ (the upper face of $R_{i+1}$ ).

- $z_{i} \rightarrow 0$, where $z_{i}$ is the $x_{n}$ coordinate of the points in the floor of $R_{i}$.

- $\left|R_{i+1}\right| \leq \alpha\left|R_{i}\right|$ for some $\alpha<1$.

- there exists a nondecreasing $C^{1}$ function $\varphi: \mathbb{R}_{\geq 0} \longrightarrow \mathbb{R}_{\geq 0}$ such that $\varphi^{\prime}$ is nondecreasing, $\varphi(0)=\varphi^{\prime}(0)=0$ and $\varphi\left(z_{i}\right)=\ell_{i}$.

Then, any $\mathcal{W}$-linked domain $\Omega$ and, more generally, any domain $\Omega$ satisfying the requirements of Remark 4.1, is called a generalized external cusp.

It is clear that a generalized external cusp $\Omega$ agrees with previous notions of external cusps. However, $\varphi$ does not give the precise profile of $\Omega$, but only a qualitative description of its narrowing toward the origin. On the other hand, the theory presented in the previous section applies straightforwardly for generalized external cusps.

In Figure 4 we show examples of general external cusps. Figure 4a is just a chain of rectangles satisfying: $L_{i+1} \sim \frac{1}{\sqrt{2}} L_{i}$ and $\ell_{i}=z_{i}^{2}$. Figure $4 \mathrm{~b}$ shows an external cusp with locally smooth 
boundary away from the origin. The interior chain of rectangles is like the one in 4a, but leant. On the other hand, Figure $4 \mathrm{c}$ is a perturbation of $4 \mathrm{~b}$, formed by a chain of locally John quasirectangles. Finally, observe that a domain satisfying (5.1), but taking $\varpi \subset \mathbb{R}^{n-1}$ a John domain with respect to the center of a cube included in $\varpi$, is a general external cusp based on John quasi-rectangles. We provide a proof of this for a particular case later. In Figure $4 \mathrm{~d}$ we present an example of this situation, taking $\varphi(t)=t^{2}$ and $\varpi$ an inner cusp like $\Omega_{\alpha}$.

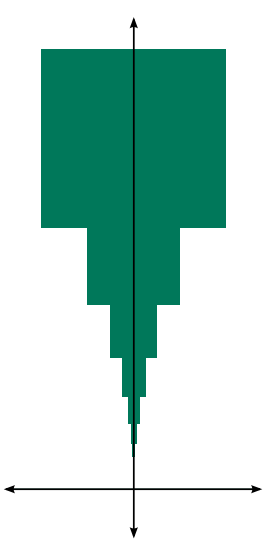

(A) External cusp using a chain of rectangles.

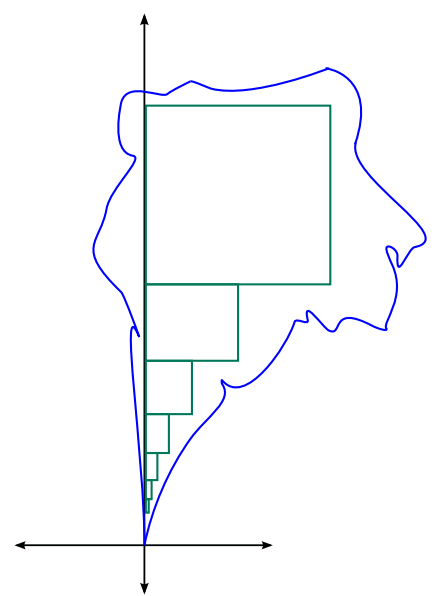

(c) Generalized external cusp based on locally John quasirectangles.

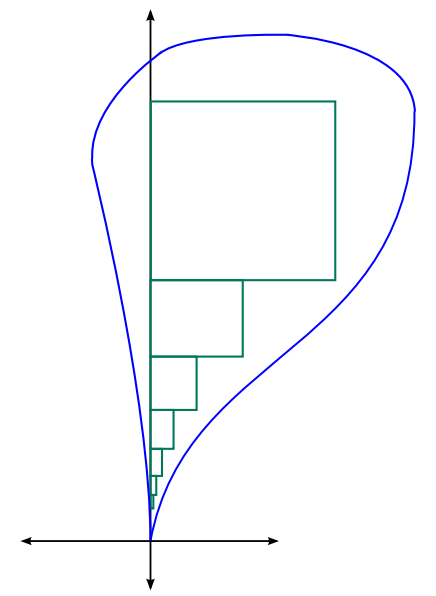

(B) A generalized external cusp.

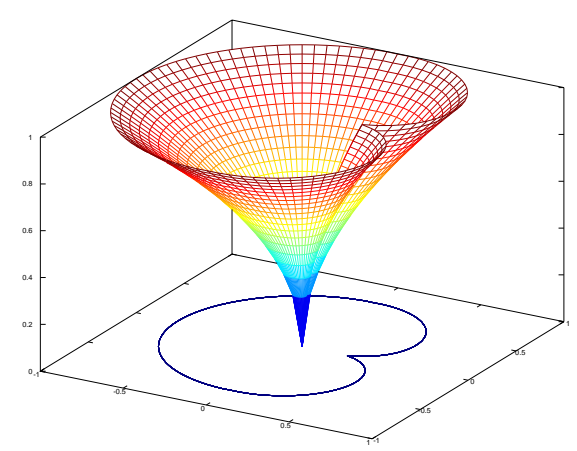

(D) Generalized external cusp by dilatation of a fixed John domain.

Figure 4. Examples of Generalized External Cusps

The unweighted results of the previous section can be immediately applied to generalized external cusps obtaining Poincaré and Korn's inequalities for them. Moreover, if we take a weight $\omega$ which is a nondecreasing function of $x_{n}$ (or $|x|$ ), we have:

$$
\omega\left(R_{i+1}\right) \leq\left\{\max _{\left[z_{i+1}, z_{i}\right]} \varphi\right\}\left|R_{i+1}\right| \leq \alpha\left\{\min _{\left[z_{i}, z_{i-1}\right]} \varphi\right\}\left|R_{i}\right| \leq \alpha \omega\left(R_{i}\right),
$$

and the decreasing property (3.4) is fulfilled. In this way we can consider some particularly interesting weights. For example, being $\varphi^{\prime}$ non-decreasing, we can take weights of the form: 
$\omega(x)=\left(\varphi^{\prime}\right)^{p \beta}$ with $\beta \geq 0$. On the other hand, we can also take weights of the form $\omega(x)=x_{n}^{p \beta}$, being $\beta \geq 0$. In this way, we obtain the following theorem where we denote $L(a)$ and $\ell(a)$ the lengths of the edges $L(R)$ and $\ell(R)$, being $R$ the rectangle at height $a$.

Theorem 5.1. Let $\Omega$ be a Generalized External Cusp, and $\sigma(x)=\left(\frac{\ell(|x|)}{L(|x|)}\right)^{-p}$. Then the inequality:

$$
\|D u\|_{L_{\omega}^{p}(\Omega)^{n \times n}} \leq C\left\{\|u\|_{L^{p}(B)^{n}}+\|\varepsilon(u)\|_{L_{\omega \sigma}^{p}(\Omega)^{n \times n}}\right\}
$$

holds for weights of the form:

$$
\omega(x)=\gamma x_{n}^{p \beta}, \quad \beta \geq 0
$$

$$
\omega(x)=\left(\varphi^{\prime}\right)^{p \beta}, \quad \beta \geq 0
$$

It is important to observe that, if $\varphi$ is such that $\varphi\left(z_{i-1}\right)-\varphi\left(z_{i}\right) \sim \varphi\left(z_{i}\right)$, then:

$$
\frac{\ell_{i}}{L_{i}}=\frac{\varphi\left(z_{i}\right)}{z_{i-1}-z_{i}} \sim \frac{\varphi\left(z_{i-1}\right)-\varphi\left(z_{i}\right)}{z_{i-1}-z_{i}} \sim \varphi^{\prime}\left(z_{i}\right) .
$$

Hence, $\sigma \sim\left(\varphi^{\prime}\right)^{-p}$ and item (b) in Theorem 5.1 is a generalization of Theorem A. In fact, as in Theorem A, the weight on the left hand side is $\left(\varphi^{\prime}\right)^{p \beta}$, whereas the one on the right hand side is $\left(\varphi^{\prime}\right)^{p(\beta-1)}$. Here, $\varphi$ is not forced to be a power function and it does not depict the precise profile of $\Omega$ but only provides a qualitative description of its cuspidal behavior, allowing the boundary of $\Omega$ to be very general considering that it might be based on locally John quasi-rectangles. It is also noteworthy that the critical case of Theorem B is reached.

On the other hand, let us consider a profile cusp satisfying (5.1), but taking $\varpi$ a John domain (an example can be seen in Figure 4d). Moreover, let us suppose $\varphi(z)=z^{\gamma}$ for some $\gamma>1$. We show how the rectangles can be chosen in order to prove that such a cusp is a generalized external cusp based on locally John quasi-rectangles.

Let us take

$$
z_{i}=\frac{1}{2^{i}}
$$

The rectangle $R_{i}$ is placed at height $z_{i}$, and the length of its edges is

$$
\ell_{i}=\varphi\left(z_{i}\right)=\frac{1}{2^{i \gamma}}, \quad \text { and } \quad L_{i}=z_{i-1}-z_{i}=\frac{1}{2^{i}} .
$$

Let us consider a weight of the form:

$$
\omega(x)=\left(\frac{\ell_{i}}{L_{i}}\right)^{p \beta}=\frac{1}{2^{i(\gamma-1) p \beta}} \quad \forall x \in R_{i} .
$$

Then

$$
\begin{aligned}
\omega\left(R_{i+1}\right) & =\frac{1}{2^{(i+1)(\gamma-1) p \beta}}\left|R_{i+1}\right|=\frac{1}{2^{(i+1)(\gamma-1) p \beta}} \frac{1}{2^{(i+1) \gamma(n-1)}} \frac{1}{2^{i+1}}=\frac{1}{2^{(i+1)((\gamma-1) p \beta+\gamma(n-1)+1)}} \\
& =\frac{1}{2^{(\gamma-1) p \beta+\gamma(n-1)+1}} \omega\left(R_{i}\right) .
\end{aligned}
$$

Hence, the decresing property (3.4) is satisfied when

$$
\frac{1}{2^{(\gamma-1) p \beta+\gamma(n-1)+1}}<1,
$$

or, in other words:

$$
(\gamma-1) p \beta+\gamma(n-1)+1>0,
$$


which leads us to:

$$
\beta>-\frac{1+\gamma(n-1)}{(\gamma-1) p}
$$

Notice that in this case $\omega \sim\left(\varphi^{\prime}\right)^{p \beta}$ and then we can express the weight in terms of $\varphi^{\prime}$.

On the other hand observe that each $R_{i}$ is a locally John quasi-rectangle. Indeed, each $R_{i}$ can be thought as an $\mathcal{W}_{i}$-linked domain, being $\mathcal{W}_{i}=\left\{\Omega_{j}\right\}_{1 \leq j \leq N}$. Here $N$ is the integer part of $\frac{L_{i}}{\ell_{i}}$ and each $\Omega_{j}$ has height almost equal to $\ell_{i}$. It can be easily seen that any $\Omega_{j}$ is a quasi-cube given by a John domain. Indeed: first show that a cylindrical set $J$ of the form $\varpi \times(a, b) \subset \mathbb{R}^{n}$, with $b-a \sim \operatorname{diam}(\Omega)$, is a John domain with constants given by those of $\varpi$. Then conclude by observing that $\Omega_{j}$ almost agrees with a dilatation of $J$ (actually it is possible to construct a bi-Lipschitz mapping $F$ such that $F\left(\Omega_{j}\right)=\ell_{i} J$, see [20]).

Therefore can now state:

Theorem 5.2. Let $\Omega$ be an external cusp satisfying (5.1), but taking $\varpi \subset \mathbb{R}^{n-1}$ a John domain, and $\varphi(z)=z^{\gamma}$, with $\gamma>1$. Then:

$$
\left.\|D u\|_{L_{\omega}^{p}(\Omega)} \leq C\left\{\|u\|_{L^{p}(B)}+\|\varepsilon(u)\|_{L_{\omega \sigma}^{p}(\Omega)}\right\}\right\}
$$

with:

being $\beta>-\frac{1+\gamma(n-1)}{(\gamma-1) p}$.

$$
\sigma(x)=\left(\varphi^{\prime}(x)\right)^{-p} \quad \text { and } \quad \omega(x)=\left(\varphi^{\prime}(x)\right)^{p \beta},
$$

This result is also a generalization of Theorem A. It imposes more restrictions than Theorem 5.1 on the boundary of $\Omega$, but it admits a negative range for the exponent $\beta$. On the other hand, the critical case $\beta_{1}=\beta_{2}+1$ in Theorem $\mathrm{B}$ is once again reached. It is important to notice that the counterexamples proposed in [14] for proving Theorem B, are given in terms of functions that depend only on the last coordinate and on the profile function $\varphi$. Consequently, they are independent of the boundary of the cusp, and can be easily adapted for general external cusps based on locally John quasi-rectangles.

Acknowledgment: We would like to thank the referee for several interesting and helpful comments.

\section{REFERENCES}

[1] Korn A. Die eigenshwingungen eines elastichen korpers mit ruhender oberflache. Akad. der Wissensch Munich, Math-phys. Kl, Beritche 1906; 36:351-401.

[2] Korn A. Ubereinige ungleichungen, welche in der theorie der elastischen und elektrischen schwingungen eine rolle spielen. Bulletin internationale, Crakovie Akademie Umiejet, Classe de sciences mathematiques et naturelles 1909; 705-724.

[3] Brenner S, Scott R. The mathematical theory of Finite Element Methods. Springer, 3rd edition, 2008.

[4] Kikuchi N, Oden JT. Contact problems in elasticity: a study of variational inequalities and Finite Element methods. Studies in Applied Mathematics. Philadelphia: SIAM, 2nd edition, 1988.

[5] Friederichs K. On the boundary-value problems of the theory of elasticity and Korn's inequality. Ann. Math 1947; 48:441-471.

[6] Nitsche J. On Korn's second inequality. RAIRO J. Numer. Anal. 1981; 15:237-248.

[7] Durán R, Muschietti MA. The Korn inequality for Jones domains. Electron. J. Diff. Eqns. 2004; 127:1-10.

[8] Jones PW. Quasiconformal mappings and extendability of functions in Sobolev spaces. Acta Math. 1981; 147(1-2):71-88.

[9] John F. Rotation and strain. Communications on Pure and Applied Mathematics 1961; XIV:391-413.

[10] Martio O, Sarvas J. Injectivity theorems in plane and space. Ann. Acad. Sci. Fenn. 1978-1979; I(4):384-401.

[11] Acosta G, Durán R, Muschietti M. Solutions of the divergence operator on John domains. Advances in Mathematics 2006; 2(26):373-401.

[12] Durán R. An elementary proof of the continuity from $L_{0}^{2}(\omega)$ to $H_{0}^{1}(\omega)^{n}$ of Bogovskii's right inverse of the divergence. Revista de la Unión Matemática Argentina 2012; 53(2):59-78. 
[13] Costabel M, Dauge M. On the inequalities of Babuška-Aziz, Friedrichs and Horgan-Payne. arXiv:1303.6141v1 2013;

[14] Acosta G, Durán R, López García F. Korn inequality and divergence operator: counterexamples and optimality of weighted estimates. Proc. Amer. Math. Soc. 2013; 141(1):217-232.

[15] Durán R, López García F. Solutions of the divergence and Korn inequalities on domains with an external cusp. Ann. Acad. Sci. Fenn. Math. 2010; 35:421-438.

[16] Nazarov SA. Notes to the proof of a weighted Korn inequality for an elastic body with peak-shaped cusps. Jour. of Math. Sci. 2012; 181(5):632-667.

[17] Acosta G, Durán R, Lombardi A. Weighted Poincaré and Korn inequalities for Hölder $\alpha$ domains. Math. Meth. Appl. Sci (MMAS) 2006; 29(4):387-400.

[18] Durán R, Muschietti MA, Russ E, Tchamitchian P. Divergence operator and poincaré inequalities on arbitrary bounded domains. Complex Variables and Elliptic Equations: An International Journal 2010; 55(8):795-816.

[19] López García F. A decomposition technique for integrable functions with applications to the divergence problem. JMMA - in press 2013; 1-19. doi:10.1016/j.jmaa.2014/03/080.

[20] Acosta G, Ojea I. Extension theorems for external cusps with minimal regularity. Pacific Journal of Mathematics $2012 ; \mathbf{2 5 9}(1): 1-39$.

[21] Kufner A, Persson LE. Weighted inequalities of Hardy type. London: World Scientific Publisher, 1st edition, 2003.

[22] Veeser A, Verfurth R. Poincaré constants for finite element stars. IMA Jour. of Num. Anal. 2012; 32(1):30-47.

[23] Hurri-Syrjänen R. An improved Poincaré inequality. Proc. Amer. Math. Soc. 1994; 120(1):213-222.

[24] Maz'ya V, Poborchiǐ S. Differentiable functions on bad domains. River Edge, NJ: World Scientific Publishing Co., 1997.

Departamento de Matemática

Facultad de Ciencias Exactas y Naturales

Universidad de Buenos Aires

PABellón I, Ciudad Universitaria

(1428) Buenos Aires

Argentina

E-mail address: gacosta@dm.uba.ar

E-mail address: iojea@dm.uba.ar 